\title{
El devenir de las instituciones educativas en el proceso de transformación social: el caso del Colegio de Bachilleres del Estado de Chihuahua, México (1973-1985) ${ }^{1}$
}

The development of the educational institutions in the social transformation process: From the High School Colegio de Bachilleres of the Chihuahua state, Mexico (1973-1985)

A evolução das instituições educacionais no processo de transformação social: o caso do Colégio de Ensino Médio do Estado de Chihuahua, México (1973-1985)

Recepción: 12/08/2019

Evaluación: 03/03/2020

Aceptación: 01/04/2020

Artículo de Investigación - Reflexión

https://doi.org/10.19053/01227238.10899

Marilyn Georgia Salcido Sáenz ${ }^{2}$ https://orcid.org/0000-0003-1091-989X

Jesús Adolfo Trujillo Holguín ${ }^{3}$ https://orcid.org/0000-0002-6738-1878

Universidad Autónoma de Chihuahua, México

\section{RESUMEN}

Objetivo: Este trabajo analiza la Chihuahua $(\mathrm{COBACH})$ en el periodo influencia de las instituciones educativas para generar transformaciones sociales a partir de la eficacia de la escuela para engendrar una cultura escolar propia. Particularmente se estudia la creación del subsistema de nivel medio superior: el Colegio de Bachilleres del Estado de de 1973 a 1985. Método: Se utiliza el enfoque histórico crítico mediante el análisis de fuentes orales y de archivo para el rescate fehaciente de los hechos. Se tomó el enfoque teórico del análisis microhistórico, pues se rescata una historia institucional regional con sentido

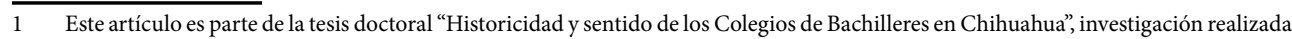
para optar por el doctorado en Educación, Artes y Humanidades de la Universidad Autónoma de Chihuahua. Esta investigación obtuvo financiamiento de las becas nacionales CONACyT.

2 Estudiante del doctorado en Educación, Artes y Humanidades de la Facultad de Filosofía y Letras de la Universidad Autónoma de Chihuahua, México. Maestra en Educación Superior y docente del Colegio de Bachilleres del Estado de Chihuahua, correo electrónico: georgiasalcido99@gmail.com,

3 Doctor en educación, docente-investigador de la Facultad de Filosofía y Letras de la Universidad Autónoma de Chihuahua, México, correo electrónico: jatrujillo@uach.mx, 
humano, basada en los testimonios de sus fundadores. Originalidad: Este estudio pretende contribuir a la investigación historiográfica educativa regional, se enfatiza en los primeros doce años del Colegio debido al crecimiento y consolidación que logró durante este periodo. Conclusiones: Los resultados evidencian cómo las instituciones educativas son capaces de engendrar culturas escolares que trascienden sus recintos y se establecen dentro de la identidad de la sociedad.

Palabras clave: Historia de la educación; Educación Media Superior; cultura escolar; planeación institucional.
This article analyzes the influence of educational institutions to conceive social transformations based on the effectiveness of the school in spawning a school culture of its own. Particularly, it is studied the creation of the upper secondary level subsystem of the Colegio de Bachilleres del Estado de Chihuahua (COBACH) from 1973 to 1985 . The present work is part of an ongoing doctoral research, which aims at the historical reconstruction of the institution. In order to develop this article, oral and archival data were used for a reliable saving of the facts. It is taken a theoretical approach of the micro-historical analysis from the rescue of the regional institutional history with a human sense, it was based on the testimonies of its founders. It is emphasized in the first twelve years of the High School because during this period it was achieved a consolidation which shows how educational institutions are able of generating its own cultures.

Key words: History of education; Middle-Upper Education; school culture; institutional planning.

\section{RESUMO}

Neste artigo se analisa a influência das instituições educacionais para promover transformações sociais a partir da eficácia da escola para criar uma cultura escolar própria. Estuda-se, de maneira particular, a criação do subsistema de nível médio superior: o Colegio de Bachilleres del Estado de Chihuahua (COBACH) no período de 1973 a 1985. O presente trabalho faz parte de uma pesquisa de doutoramento em curso, cujo objetivo é a reconstrução histórica da instituição. Para o desenvolvimento deste artigo, fez-se uso de fontes orais e de arquivo para um resgate fidedigno dos fatos. $\mathrm{O}$ enfoque teórico é a análise micro-histórica, pois se resgata uma história institucional regional com sentido humano, baseada nos testemunhos de seus fundadores. Recorte temporal são os primeiros doze anos do Colégio, devido ao crescimento e consolidação que alcançou neste período, o que evidencia como as instituições educacionais são capazes de engendrar culturas escolares que transcendem seus espaços e se estabelecem dentro da identidade da sociedade.

Palavras-chave: História da educação; Ensino Médio Superior; cultura escolar; planejamento institucional. 


\section{INTRODUCCIÓN}

En la historia de la educación del Estado de Chihuahua hay ausencia de trabajos que daten el inicio y la consolidación del $\mathrm{COBACH}$, el cual opera en la actualidad, y desde el momento de su creación, como el mayor subsistema de nivel medio superior en la entidad, y de ello se deriva que sea la institución con el mayor número de jóvenes egresados de la Educación Media Superior (EMS).

Como punto de partida, la falta de trabajos de investigación sobre el tema da pertinencia al desarrollo del presente artículo. Propiamente existe información insustancial y de carácter informativo en la página web de la institución. Además, se han realizado estudios que analizan el surgimiento de este centro educativo pero desde la capital del país, aludiendo a que este organismo empezó en la ciudad de Chihuahua sin profundizar en ello. Inclusive, recientemente se publicó un libro que narra la historia del Plantel 5 ubicado en Ciudad Juárez, el cual sería el primero en crearse en dicha ciudad y ofrece una breve reseña de los antecedentes del Colegio. Si bien el presente artículo se apoya en dicha información, para darle rigurosidad científica contrasta la veracidad de la misma con la indagación en otras fuentes. A causa de ello, este trabajo pretende figurar como la primera aportación a la historia de esta institución en sus primeros años, además de contribuir a la historia regional. Es importante señalar que el $\mathrm{COBACH}$, a pesar de que fue un proyecto que se realizó en el ámbito nacional, tuvo sus primeros planteles en el Estado de Chihuahua debido al cierre de la Escuela Preparatoria de la Universidad Autónoma de Chihuahua (UACH).

El estudio tiene por objeto analizar y comprender los acontecimientos que motivaron la creación del Colegio, acudiendo a la memoria de los sujetos para hacer su reconstrucción histórica. Se pretende profundizar en la historia de esta institución no solo de una manera genealógica, sino para comprender el porqué y el cómo de su instauración, su progreso, su evolución y su permanencia como la opción número uno de ingreso al nivel medio superior.

Asimismo, se busca aportar a la identidad institucional del Colegio, pues resulta significativo reconocer toda su significativa trayectoria para el sistema educativo, así como para la sociedad chihuahuense. Y hablar de identidad compromete a manifestar qué se es y qué no se es, pues se erige una forma de representar a la institución para la sociedad y para sus actores. Es importante destacar que la identidad que se detalla va acompañada por la temporalidad del estudio, no es una concepción determinada y terminal. Por el contrario, muestra la naturaleza de la identidad contradictoria, histórica y diferencial ${ }^{4}$.

Para la realización de este trabajo se utilizó el enfoque metodológico histórico crítico, se manejó la técnica de historia oral para recolectar información en fuentes primarias, las cuales se centraron en entrevistas semiestructuradas a docentes y alumnos fundadores de dicha institución. Al considerarse la temporalidad del estudio como parte de la historia reciente, resulta valioso el rescate de la información por medio de los testimonios debido a que es posible profundizar en las experiencias de los participantes y permite, al mismo tiempo, adentrarse en el contexto donde emergió esta institución.

4 Zaira Navarrete Cazales, “¿Otra vez la identidad? Un concepto necesario pero imposible”, Revista Mexicana de Investigación Educativa Vol. 20, n. ${ }^{\circ} 65$ (2015): 473-474. 


\section{Antecedentes}

El COBACH es una institución de Educación Media Superior que se creó por decreto presidencial el 19 de septiembre de 1973 en la ciudad de Chihuahua, bajo la gestión del presidente Luis Echeverría Álvarez, esto debido al cese de la Escuela Preparatoria de la Universidad ${ }^{5}$.

El modelo nacional de bachillerato general Colegio de Bachilleres, inició en la ciudad de Chihuahua para sosegar el enfrentamiento que existía en la universidad debido a los levantamientos y conflictos políticos que surgieron a raíz del movimiento universitario de 1968. El proyecto de creación de los Colegios de Bachilleres fue orientado para iniciar labores en la Ciudad de México en 1974; sin embargo, debido a la situación que en aquellos años albergaba la capital del Estado, se vio la necesidad de llevarlo a cabo en la ciudad de Chihuahua ${ }^{6}$.

Actualmente el COBACH se constituye como el más grande subsistema de EMS en la entidad, cuenta con 31 planteles en las modalidades escolarizada y no escolarizada, y se sostiene como la opción número uno de ingreso al nivel medio superior por los jóvenes chihuahuenses ${ }^{7}$.

\section{La cultura escolar en su perspectiva histórica}

El espacio escolar se define como un recinto que engendra una cultura propia, el cual, mediante las prácticas que ejerce bajo un esquema axiológico, persigue educar a un proyecto de ser humano. Este ideario de persona se construirá con base en un perfil ideológico que estará determinado por un modo organizativo y una intencionalidad; particularmente la educación formal está condicionada por el espacio, el tiempo, el lenguaje y el discurso que se maneja ${ }^{8}$. La expresión de espacio escolar también se le denomina cultura escolar, la cual está constituida:

Por un conjunto de teorías, ideas, principios, normas, pautas, rituales, inercias, hábitos y prácticas - formas de hacer y pensar, mentalidades, comportamientossedimentadas a lo largo del tiempo en forma de tradiciones, regularidades y reglas del juego, no puestas en entredicho y compartidas por sus actores en el seno de las instituciones educativas. ${ }^{9}$

La cultura escolar que engendra el COBACH se apropia de un espacio inmaterial que será transmitido de generación en generación, perdurando más allá

\footnotetext{
5 Colegio de Bachilleres del Estado de Chihuahua (COBACH), Quiénes somos (Chihuahua: 2018), http://www.cobachih.edu.mx/ Colegio/Con\%C3\%B3cenos/Rese\%C3\%B1a-Hist\%C3\%B3rica (1. ${ }^{\circ}$ de febrero, 2019).

6 Alma Rosa Neyra Galicia, "El bachillerato mexicano y la política educativa: desde sus inicios hasta la educación basada en competencias", Textual. Análisis del Medio Rural Latinoamericano, n. ${ }^{\circ} 55$ (2010): 68.

7 COBACH, Quiénes somos (Chihuahua: 2018).

8 Antonio Viñao Frago, “El espacio escolar: introducción”, Historia de la Educación, n. ${ }^{\circ} 12$ (1993): 14.

9 Antonio Viñao Frago, "Culturas escolares, reformas e innovaciones educativas", Con-ciencia social, n. ${ }^{\circ} 5$ (2001): 31.
} 
de los cambios para el progreso de la institución. Por lo tanto, el quehacer del historiador será esbozar esta cultura escolar en los aspectos más ostensibles que la configuran.

De entrada, debemos situarnos en los precedentes causales que dieron inicio a esta institución. Entre los años de 1968 y 1973, existió en México un fuerte descontento por parte de los estudiantes con las autoridades políticas y educativas a partir de los acontecimientos de Tlatelolco. Específicamente para el Estado de Chihuahua estalló una pugna entre los estudiantes y la universidad, con la Escuela Preparatoria - encargada del nivel medio superior en la localidad - ubicada como foco de conflicto. Se configuraba como un centro de colisión por el hecho de que era la escuela que albergaba más jóvenes dentro de la universidad, además de ser los más maleables para realizar movimientos y atracos en contra de la autoridad ${ }^{10}$.

Imagen 1. Entrada principal de la Escuela Preparatoria de la UACH vandalizada por los estudiantes.

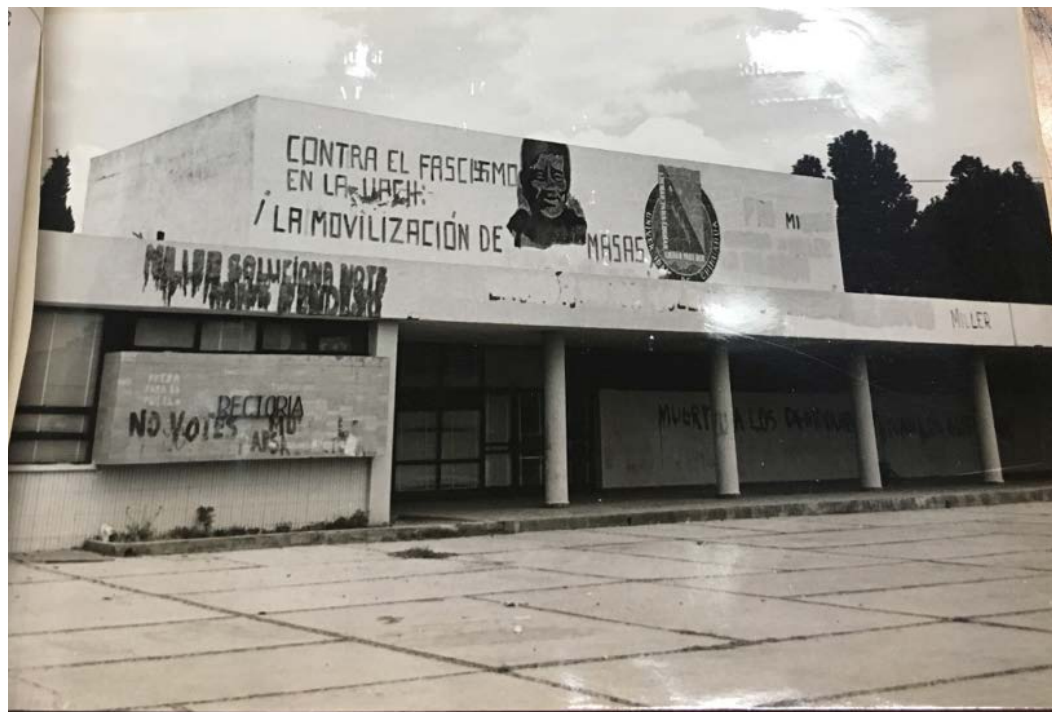

Fuente: Carta notariada certificada por el Lic. José A. Molina que da fe mediante las fotografías de los destrozos ocasionados en la Escuela Preparatoria (Fondo UACH, en AHUACH).

El cierre de la Escuela Preparatoria se dio en 1973 y ocurrió cuando los estudiantes realizaron un levantamiento que terminó en daños severos de vandalismo para la institución (imagen 1). Paredes exteriores pintadas, vidrios rotos, puertas vencidas, murales destruidos por disparos de armas de fuego de diferentes calibres, destrozos a la casi totalidad del mobiliario de la institución (mesas, bancas, aparatos telefónicos, archiveros, sanitarios, entre otros);

10 Entrevista a Rico Bovio, Arturo, Chihuahua, 20 de febrero de 2018. 
además, en la sala de música se encontró un piano de cola totalmente destruido (imagen 2). Se hallaron rastros de fogatas prendidas dentro de las instalaciones, se encontraron mensajes en los muros del exterior como: Contra el fascismo de la UACH: ¡la movilización de masas!, desarrollemos el poder político-militar del proletariado ${ }^{11}$. Este último levantamiento por parte de los estudiantes dio fundamento suficiente a las autoridades para clausurar la Preparatoria y así poder formar una nueva institución libre de ideologías bajo un nuevo régimen. El cese de la Escuela Preparatoria se dio en 1974 lo que desencadenó "el despido de decenas de catedráticos y la suspensión de los derechos estudiantiles. Este proceso representó la pérdida del legado del pensamiento crítico, democrático y humanista, así como la supresión de la Educación Media Universitaria Pública en el estado"12. Debido a que, "la finalidad primordial era crear un nuevo sistema de nivel medio superior emancipado de la educación universitaria, que diera respuesta a la demanda de espacios educativos y que acabara de tajo con el problema en la Escuela Preparatoria"13.

Imagen 2. Piano de cola destruido.

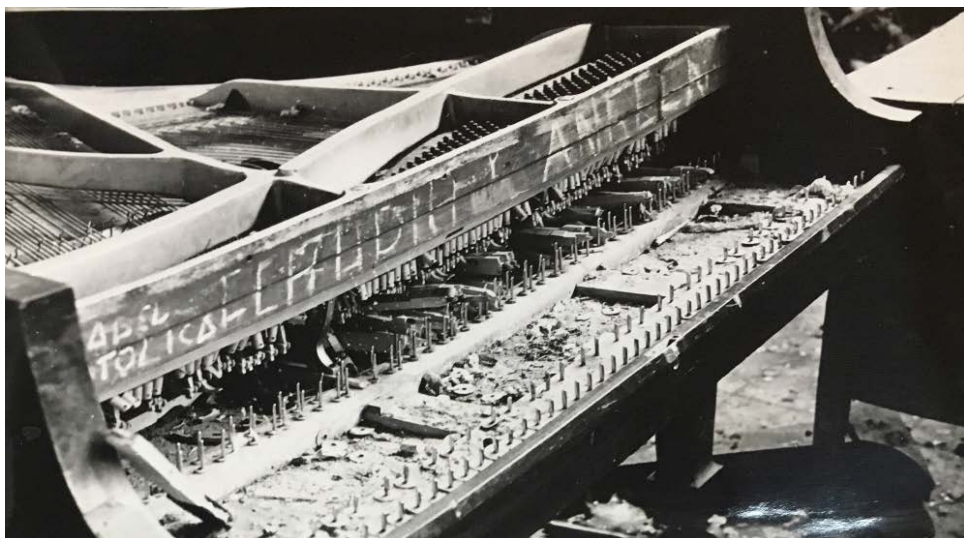

Fuente: “Carta notariada certificada por el Lic. José A. Molina que da fe mediante las fotografías de los destrozos ocasionados en la Escuela Preparatoria". (Fondo UACH, en AHUACH).

La creación del $\mathrm{COBACH}$ se constituye como una transformación histórica, dado que, a través de su instauración, surgieron cambios sustanciales en las prácticas educativas de la época y en los discursos que se llevaban a los estudiantes. Sassen lo define como "un nuevo ensamblaje de territorio, autoridad

11 "Carta notariada certificada por el Lic. José A. Molina" (Chihuahua, México, 1974). Archivo Histórico de la Universidad Autónoma de Chihuahua (AHUACH), Fondo UACH.

12 Gerónimo Ontiveros Juárez, Francisco Alberto Pérez Piñón y Jesús Adolfo Trujillo Holguín, "El pensamiento crítico, democrático, humanista y el movimiento estudiantil de la UACH (1968-1974)", Millcayac. Revista digital de Ciencias Sociales Vol. VII, n. ${ }^{\circ} 12$ (2020): 360 .

13 Marilyn Georgia Salcido Sáenz y Jesús Adolfo Trujillo Holguín, "La construcción de procesos de identidad en las comunidades educativas, el caso del Colegio de Bachilleres del Estado de Chihuahua”, Debates por la Historia Vol. VIII, n. ${ }^{\circ} 1$ (2020): 251. 
y derechos constituido mediante una lógica organizadora que difiere de lo anterior, aunque se apropia de algunas de sus capacidades ${ }^{14}{ }^{14}$, es decir, siendo el colegio sucesor de la Escuela Preparatoria de la UACH, ocuparía el mismo lugar social que tenía la institución desaparecida. El COBACH acogería aquellos aspirantes que tenían pensado ingresar a la preparatoria, sin embargo, la opción pública de ingreso a la EMS era ahora el colegio.

La clausura de las escuelas preparatorias adscritas a las universidades fue algo que sucedió a escala nacional debido a varias razones, una de las principales era separar la EMS de la superior y así eliminar el aval automático de ingreso a los estudiantes a la educación superior por ser parte de la misma institución. Además, se buscaba otorgar al nivel medio superior un carácter terminal en una modalidad de tres años que dotaría a los estudiantes de conocimientos básicos sobre un campo de formación para el trabajo, de manera que pudieran elegir entre continuar los estudios profesionales o ingresar al sector productivo ${ }^{15}$.

Desde los primeros conflictos suscitados en la Escuela Preparatoria se divulgaba en todo México la noticia de la clausura de las escuelas preparatorias universitarias para ser sustituidas con la fundación del sistema Colegio de Bachilleres a lo largo del país. Debido a esta premisa, un grupo de consejeros universitarios pertenecientes a la UACH -junto con el rector, el Lic. Óscar Ornelas Küchle, apoyados por el gobernador, el Lic. Óscar Flores Sánchez- realizaron un viaje a la Ciudad de México para pedir al presidente, el Lic. Luis Echeverría Álvarez, el llevar adelante esta institución en la ciudad de Chihuahua, para así poder dar cese a la Escuela Preparatoria de la UACH. Dada la autorización del primer mandatario de la nación, se empezó la planificación del proyecto Colegio de Bachilleres sin tener la viabilidad de recursos ni las instalaciones adecuadas.

Se organizaron reuniones con gente que pudiera contribuir a configurar esta nueva institución, en especial con maestros que trabajaban en la antigua preparatoria por su conocida trayectoria en la EMS. Se tuvo el cuidado de entrevistar a maestros que manejaran un perfil alejado de la ideología izquierdista y que estuvieran comprometidos con el nuevo proyecto educativo y social que se quería para la juventud. Aunque hubo contrataciones de maestros conocidos por militar en el Partido Popular Socialista (PPS) o por su simpatía con las ideas de izquierda, en particular los maestros normalistas, no se aceptaron personas que desvirtuaran su labor docente organizando revueltas o atrayendo al alumnado a la política.

En dichas reuniones se hablaba principalmente de las condiciones de trabajo, de la falta de recursos para pagos a docentes, al menos durante los primeros

14 Saskia Sassen, Territorio, autoridad y derechos. De los ensamblajes medievales a los ensamblajes globales (Buenos Aires: Katz, 2010), 31.

15 Lorenza Villa Lever, “La educación media superior: su construcción social desde el México independiente hasta nuestros días”, en Los grandes problemas de México: VII Educación, eds. Alberto Arnaut y Silvia Giorguli (México: El Colegio de México, 2010) 285. 
meses, hasta que la federación mandara recursos, además de la ausencia de programas de estudio con los cuales empezar ${ }^{16}{ }^{17}$.

La opción que se dio a los estudiantes de la antigua Escuela Preparatoria para concluir sus estudios fue acreditar, mediante exámenes, las asignaturas que les habían quedado pendientes al momento del cierre. Como las instalaciones de la preparatoria estaban tomadas y por lo tanto resultaba imposible acceder a ellas, la aplicación de estos exámenes se llevó a cabo en diferentes predios de otras escuelas ${ }^{18}$.

Imagen 3. Anuncio de la convocatoria para la recepción de documentación del Colegio de Bachilleres.

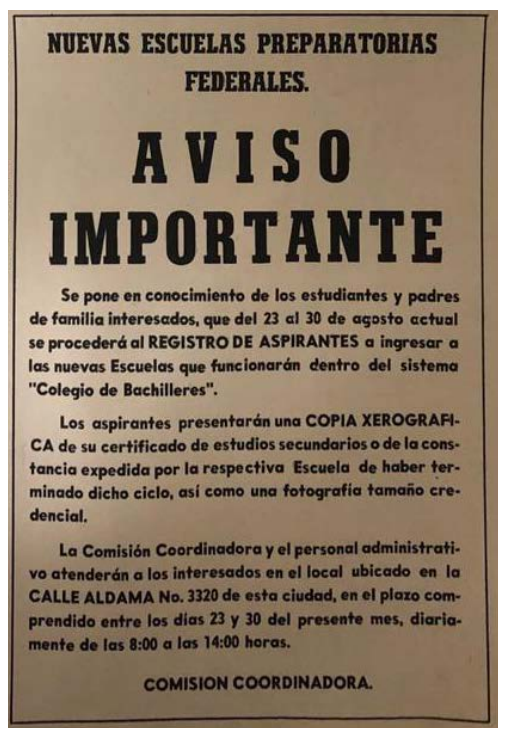

Fuente: El Heraldo de Chihuahua, lunes 27 de agosto de 1973.

Para el proceso de ingreso al $\mathrm{COBACH}$, divulgado a través de una convocatoria publicada por los diferentes periódicos (imagen 3), se invitaba a los aspirantes a registrar su documentación en el domicilio señalado, donde los futuros docentes de la institución se encargaban de recibir dicha papelería e informaban a los estudiantes sobre el proceso a seguir (imagen 4). Posteriormente se realizó un examen tipo consulta que en esencia tenía como objetivo conocer datos personales del alumno y sus inclinaciones ideológicas. En las listas de aceptados que se publicaban al final del proceso los estudiantes eran distribuidos por sectores de acuerdo con la ubicación de su domicilio. Después se publicaron las listas de aceptados por cada plantel, en donde se cuidó ubicar por sector territorial a los estudiantes ${ }^{19}$.

\footnotetext{
16 Entrevista a Duarte Huerta, Rodolfo, Chihuahua, 24 de septiembre de 2018.

17 Entrevista a Cendón Chávez, Gloria, Chihuahua, 14 de noviembre de 2018.

18 Entrevista a Duarte Huerta.

19 Entrevista a García Quintana, Raymundo, Chihuahua, 14 de marzo de 2018.
} 
Imagen 4. Recepción de documentos para ingresar al Colegio de Bachilleres.

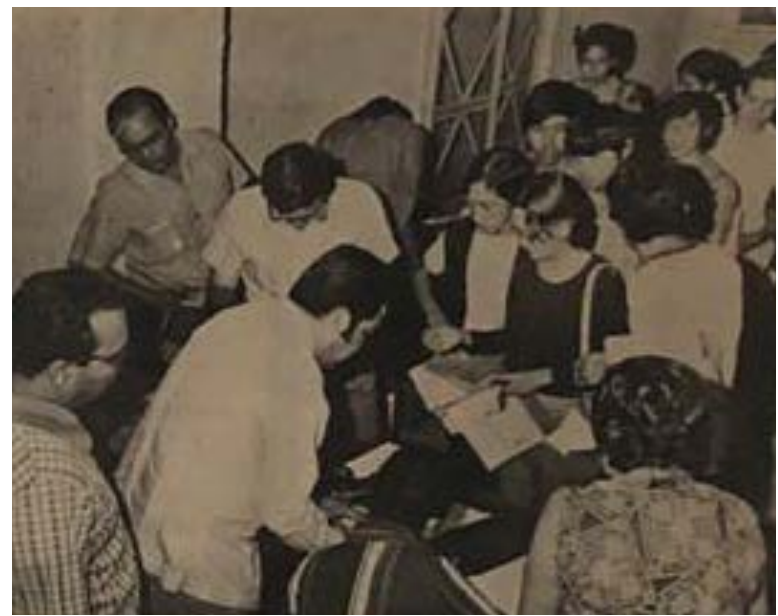

Fuente: El Heraldo de Chihuahua, viernes 24 de agosto de 1973.

Si bien la creación del COBACH aparecía como una respuesta rápida y sin una planificación bien definida para llenar el hueco que había dejado la antigua preparatoria, era la opción que complacía a la perfección los nuevos intereses de las autoridades gubernamentales y educativas. Pues aun en la austeridad del arranque del proyecto, se planteó un conjunto de reglas mediante las cuales se buscaba encaminar a la juventud hacia nuevos ideales de vida. La disciplina intolerante que imperó durante los primeros años del colegio tuvo una influencia determinante para la construcción de la cultura escolar de la institución. La educación formal que inculcaría el COBACH, sería "una apuesta por la transformación de orden ontológico, axiológico, filosófico, pedagógico, organizacional, curricular, administrativo, relacional y de la interacción humana en contextos escolares y sociales" 20 .

El COBACH se estableció como un engranaje educativo orientado hacia las nuevas ideas de progreso en el marco de una sociedad globalizada que requería de sujetos socialmente adaptados para insertarse en un mundo industrial, lo cual daría inicio a un nuevo "fenómeno constitutivo de la nueva lógica organizadora" ${ }^{21}$. La importancia que tendría el COBACH en los aspectos ideológicos, los conflictos y la valoración de los centros educativos como focos de decisión y poder, sería trascendental para la nueva configuración que tendría esta institución. La autoridad tomó a "la educación como una herramienta para forjar una cultura política acorde al régimen que se buscaba construir, valorizando

20 Yeison Arcadio Meneses Copete, "La etnoeducación afrocolombiana: concepto, trabas, patriarcado, y sexismo. A propósito de los 20 años de la Ley General de Educación 115 de 1994”, Revista Historia de la Educación Latinoamericana, Vol. 18, n. ${ }^{\circ} 27$ (2016): 64.

21 Sassen, Territorio, autoridad y derechos. 
discursos nacionalistas que evolucionaron con el cambio de los proyectos políticos" 22 .

El esbozo de la cultura escolar para la planificación de la red educativa del Colegio de Bachilleres fue totalmente cuidadosa en cuanto al nuevo proyecto de juventud que se quería producir. Debido a las razones sociales y políticas suscitadas al momento del cierre de la preparatoria, para la configuración del colegio se optó por crear varios planteles que distribuyeran el alumnado por zonas, dado que uno de los problemas de organización que realzaba los conflictos en la antigua escuela era la concentración de los estudiantes en un mismo recinto.

El COBACH, aunque inició en la ciudad de Chihuahua, durante sus primeros años se manejó como una coordinación sectorial, la cual dependía totalmente del centro del país. Desde la Ciudad de México se mandaban recursos y los planes de estudio. Este modelo de gestión operó bien en sus primeros años debido a que se manejaban pocos planteles y poca gente, por lo que el trabajo se generaba de una manera familiar. Es común escuchar que el colegio se constituyó como una gran familia pues la cercanía que existía entre los trabajadores generaba ese ambiente ${ }^{23}$.

El primer encargado de la coordinación sectorial, y fundador de la institución, fue el Lic. Óscar Ornelas Küchle, quien anteriormente se había desempeñado como rector de la universidad, cargo del cual fue cesado debido a las protestas y movimientos estudiantiles suscitados en aquellos años. No obstante, Ornelas Küchle fue para el colegio un hombre emblemático y visionario que, caracterizado por su gran vocación académica, brindó solidez y estabilidad a ese nuevo proyecto y lo encaminó hacia las sendas de la exigencia académica. Ornelas llevó a cabo su gestión de la coordinación sectorial de 1973 a 1974, con el apoyo de los licenciados Sergio Martínez Garza y Juan Meouchi. Posteriormente, el Lic. Martínez Garza asumió la dirección general de la coordinación de 1974 a $1985^{24} 25$.

22 Elías Pizarro Pizarro y Raúl Bustos González, "Educación y control político-social del Estado: visitadores de escuela en Tacna y Arica (1880-1900)", Revista Historia de la Educación Latinoamericana, Vol. 17, n. ${ }^{\circ} 25$ (2015): 127.

23 Entrevista a Cendón Chávez.

24 Agustín García, “Colegio de Bachilleres, a 45 años de su fundación”, El Heraldo de Chihuahua, Chihuahua, 17 de abril de 2018 , https://www.pressreader.com/mexico/el-heraldo-de-chihuahua/20180417/281788514651041

25 Lombardo Hagelsieb Lerma, Inicio de la era del Colegio de Bachilleres en nuestra frontera. Plantel N5 (Ciudad Juárez: 2018 ), 31. 
Imagen 5. Instalaciones del Plantel 1, edificio que anteriormente ocupaba el Instituto Regional.

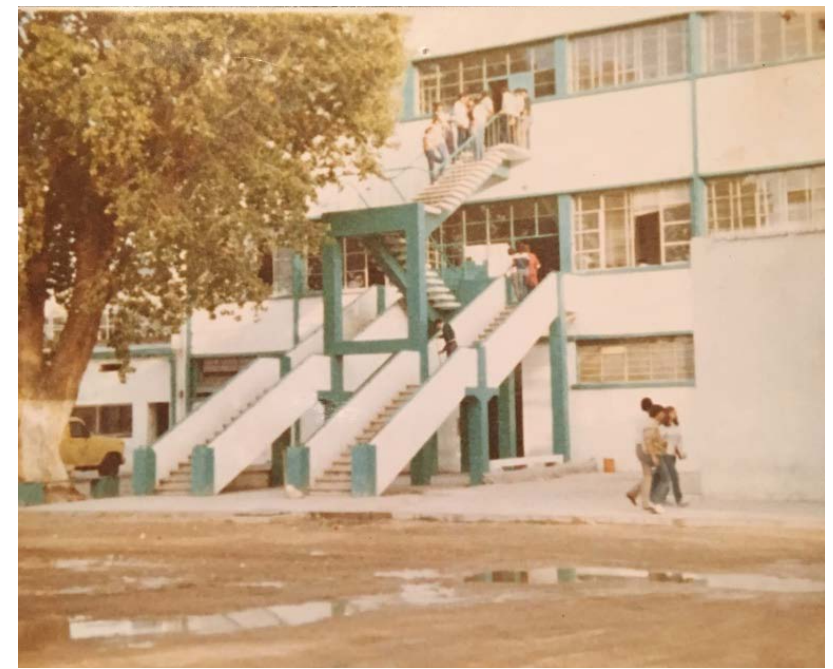

Fuente: Archivo personal de Dora Elena López López.

El COBACH inició labores con tres recintos. El Plantel 1 empezó a funcionar en las instalaciones de una antigua escuela primaria ubicada en la calle $18^{\underline{a}}$ y Jiménez; posteriormente, en 1977, con apoyo del gobierno se autorizó la construcción del plantel, adquiriendo las antiguas instalaciones del Instituto Regional (imagen 5) ubicado en la avenida Cuauhtémoc 1609 -donde se ubica actualmente-, bajo la gestión del director, Prof. Roberto Pacheco Peña, el subdirector del turno matutino, C. P. Gonzalo Alberto Aguilera Gutiérrez y el subdirector del turno vespertino, Prof. Ernesto Madrid Barroso. El Plantel 2 tuvo su primera ubicación en las calles Vicente Guerrero y Coronado, y en 1975 se mudó a la avenida Simón Bolívar e Independencia y después a la calle 9a. Para 1979 se inició la construcción del plantel en su ubicación actual, en las instalaciones de la calle $12^{\mathrm{a}}$, entre las calles J. J. Calvo y $10^{\mathrm{a}}$, bajo la gestión del director, Ing. Raúl Medrano Morales, y los subdirectores, el Ing. José Manuel Vázquez García y el Ing. Agustín Romo Duarte. Por último, el Plantel 3 tuvo su primer domicilio en las calles $35^{\mathrm{a}}$ y Aldama, después se cambió a las instalaciones donde se ubicaba el Instituto Femenino de Chihuahua en la avenida Heroico Colegio Militar y en 1976 se iniciaron los trabajos para la construcción del plantel en su ubicación actual, en el bulevar Antonio Ortiz Mena 314, bajo la gestión del director Ing. 
Víctor Martínez Testa y los subdirectores, el Prof. Héctor Hagelsieb Lerma y el Ing. Leonel Arzaga Ávila (imagen 6) 262728293031.

Los tres planteles laboraban en turnos matutino y vespertino, a excepción del Plantel 2, que además atendía en turno nocturno a los alumnos trabajadores que necesitaban un horario alterno para concluir sus estudios de bachillerato. Como el turno nocturno se manejaba en la antigua Escuela Preparatoria, el COBACH ofreció igualmente este servicio para saciar la necesidad que existía de brindar la educación media a este sector de la población ${ }^{32}$.

Imagen 6. Maestros fundadores del Plantel 3, turno vespertino

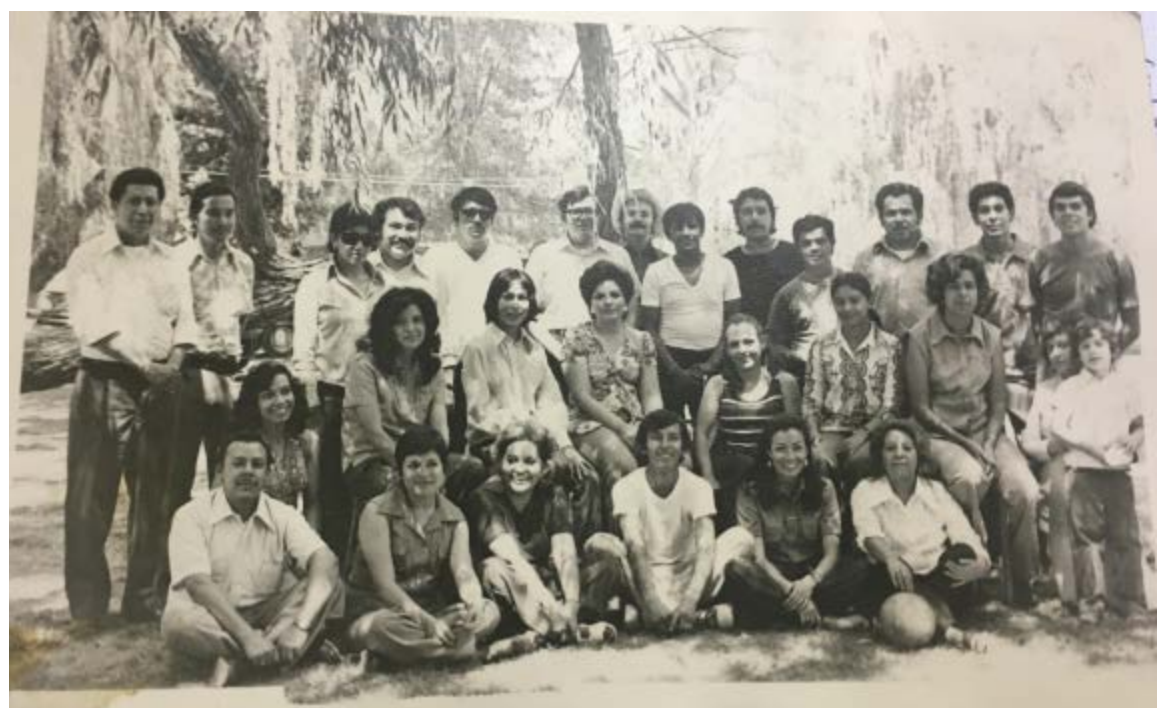

Fuente: Archivo personal de Olaya Morán.

El contexto escolar que tuvo en sus primeros años el colegio - refiriéndonos a este espacio como un lugar - era un ambiente de penuria material e instrumental debido a que las primeras instalaciones de cada plantel eran viejas y deterioradas casas de habitación, las cuales se trataron de acondicionar lo mejor posible para el ámbito educativo. A pesar de la envejecida infraestructura, allí se consiguió propiciar un ambiente escolar que favoreciera los procesos de enseñanza y aprendizaje. La consciencia entre el alumnado del gran compromiso y vocación de sus profesores - que trabajaban en una difícil situación de inestabi-

26 COBACH, Quiénes somos (Chihuahua: 2018).

27 COBACH, Historia (Chihuahua: 2018), http://cobachih.edu.mx/Planteles/Chihuahua/Plantel-1 (3 de febrero, 2019).

28 Ibíd., http://cobachih.edu.mx/Planteles/Chihuahua/Plantel-2 (4 de febrero, 2019).

29 Ibíd., http://cobachih.edu.mx/Planteles/Chihuahua/Plantel-3 (5 de febrero, 2019).

30 García, "Colegio de Bachilleres".

31 Entrevista a Cendón Chávez.

32 Entrevista a Duarte Huerta. 
lidad económica por no tener un sueldo fijo-, generaba un deseo de superación y aprovechamiento, y el sentimiento de que no podían más que retribuirlos con buenas conductas y un buen desempeño ${ }^{33}$.

Como parte de la estructura administrativa del colegio, fundamentada en lineamientos tomados de la Escuela Normal, se creó el departamento de psicopedagogía, conocido actualmente como el departamento de control escolar, el cual hacía seguimiento a cada estudiante mediante un kardex donde se registraban, de manera manual, los datos de los estudiantes y el historial de calificaciones ordinarias y extraordinarias a lo largo de cada semestre. Además, se vio la necesidad de complementar la formación profesional de los docentes del $\mathrm{COBACH}$ con un curso propedéutico de conocimientos psicológicos y pedagógicos. Los docentes del colegio procedían de una carrera universitaria que les brindaba los conocimientos técnicos de la asignatura, pero carecían de los conocimientos pedagógicos básicos para enseñar, por lo que el curso se tomaba de manera obligatoria por todos los docentes que ingresaban al servicio y posteriormente complementaban con cursos de mejora continua para capacitación de los trabajadores $^{34}$.

La transformación social que surge a raíz de la creación del $\mathrm{COBACH}$ se suscita en la nueva configuración del colegio, la cual estaría determinada por las nuevas prácticas y rituales, el ritmo de la clase, los métodos disciplinarios e instructivos y el discurso llevado a los estudiantes. Todo ello generaría la cultura escolar de la institución, blindada a través de los muros de sus espacios educativos con nuevas formas de hacer y de pensar ${ }^{35}$.

El COBACH se definió históricamente según los propósitos, valores y normas que socialmente eran necesarios para la juventud de esos años; los directivos y docentes fundadores consideraban totalmente necesaria la disciplina y el orden, pues la función social bajo la cual definían a los espacios educativos era reproducir formas de pensar, valores y comportamientos; sin embargo, para el colegio, en primera instancia, no había que reproducir sino provocar una transformación social para poder producir un nuevo sujeto que estuviera alejado de todas las agitaciones de la época.

El colegio, explícitamente, no formaba a sus estudiantes bajo una ideología específica, pues no se les obligaba a tener filiación política o creencia religiosa; sin embargo, todas las prácticas que se llevaban a cabo reprendían severamente actitudes de izquierda o cualquier manifestación que pudiera incidir en un movimiento estudiantil, el derecho de réplica contra la autoridad y los grupos de debate, conductas comunes en la antigua preparatoria. Aunque no se considerara que se educaba bajo cierta tendencia, en realidad, de una forma inadvertida -incluso para el personal docente- se iba inclinando a la juventud mediante normas, códigos y rituales hacia actitudes que manifestaran el mantenimiento

33 Entrevista a García Quintana.

34 Entrevista a López López, Dora Elena, Chihuahua, 26 de octubre de 2018.

35 Antonio Viñao Frago, "Del espacio escolar y la escuela como lugar: propuestas y cuestiones", Historia de la Educación, n. ${ }^{\circ}$ 12-13(1993): 27. 
de las jerarquías, la defensa de la tradición nacionalista y el control sobre la organización social, en este caso estudiantil. Particularmente, los docentes concebían los modos de educar del colegio como una manera de formar buenos mexicanos que velaran por el bien del país ${ }^{36}$.

Los dirigentes del COBACH concebían la utilidad de la organización social que brindaban los espacios educativos - el ejercicio del poder que ofrecían los recintos era inmejorable - pues se podían institucionalizar las dinámicas y prácticas que el colegio llevaba a cabo. Estas prácticas institucionalizadas constituyeron un proceso histórico tanto para la escuela como para la sociedad chihuahuense, puesto que la aprobación que la sociedad dio a las mismas constituyó un modo propio de aceptación y rutinización. Es importante mencionar que no fue fácil implementar las prácticas y dinámicas que configuraron lo que hoy es el colegio; uno de esos cambios significativos, que se fueron dando paulatinamente, fue la obligatoriedad del uniforme, cuyo uso en un principio no se implementó como una imposición, aunque sí existía una reglamentación estricta sobre el código de vestimenta de los estudiantes, así como de su aseo personal. "Los alumnos debían calzar zapato formal, no deportivo o sandalias, debían andar fajados, las mujeres no podían vestir minifaldas o blusas con tirantes, para los hombres el cabello corto era obligatorio" ${ }^{37}$. Pasaron alrededor de ocho años para que se institucionalizara el uniforme y se hizo por medio de un proceso democrático, bajo el consentimiento de los padres de familia.

El colegio se caracterizó en sus primeros años por ser un referente de excelencia académica, si bien los planes de estudio eran enviados por la coordinación en la Ciudad de México. En estos años iniciales se utilizaron como base los programas que se manejaban en los Colegios de Ciencias y Humanidades $(\mathrm{CCH})$ de la capital de país. Asimismo, gracias al entusiasmo y la dedicación de los docentes hacia la institución, se hacía un análisis de estos programas para realizar adaptaciones de acuerdo con el nivel que se trabajaba y de manera colegiada - en horario extra laboral - se conformaban los programas de cada asignatura, cuidando siempre que los contenidos fueran los adecuados para brindarle al estudiante las herramientas necesarias para el ingreso a la educación superior. "La institución por muchos años mantuvo los primeros lugares en los exámenes de ingreso a la Universidad incluso por arriba de instituciones privadas" ${ }^{\prime \prime 3}$.

El COBACH también se distinguió por lograr un éxito incomparable a los demás colegios que se abrieron en las distintas entidades federativas. Particularmente, en los otros estados de la República los Colegios de Bachilleres figuraron como una opción más para cursar la EMS. No obstante, para la ciudad de Chihuahua el colegio fue la primera opción de ingreso y hasta la fecha se ha mantenido de ese modo ${ }^{39}$, siempre respaldado por la excelencia académica

\footnotetext{
36 Entrevista a López López.

37 Entrevista a Cendón Chávez.

38 Ibíd.

39 Entrevista a Duarte Huerta.
} 
que lo distingue, cumpliendo además con el propósito transformador para la sociedad con el que la institución fue creada para esta ciudad. La reglamentación académica fue muy estricta en sus inicios con la finalidad de hacer entrever que el objetivo de la institución era educar y formar jóvenes comprometidos con su educación y con la sociedad. "Los alumnos con reprobar una sola materia estaban sujetos a la expulsión del Colegio" ${ }^{40}$.

Los tres planteles que se crearon en la ciudad de Chihuahua para dar inicio al Colegio de Bachilleres tenían la característica de albergar a estudiantes de todas las clases sociales, algunos más concentrados en un sector de la población que otros por la zona territorial en la que se encontraban situados, pero absorbiendo al fin de cuentas a una comunidad heterogénea en cuestión socioeconómica ${ }^{41}$. A causa de ello, la cultura escolar generada por la institución se propagó a todos los niveles de la población y, debido a ello, el proceso de transformación social no tuvo límites, lo que produjo una metamorfosis total.

Por su carácter público, la formación educativa que brindaría el sistema COBACH debía cubrir aspectos básicos para su configuración y sería, primeramente, de carácter formal y gestionada por un agente público. En sus primeros años ese agente fue la federación, pues se había constituido como una organización centralizada, dirigida por el Colegio de Bachilleres de la Ciudad de México (COLBACH), el cual formaba parte de su estructura orgánica que, a su vez, estaba financiada en su mayoría por la administración pública, considerando que el colegio en su inicio no contaba con recursos suficientes para su sostenimiento. Asimismo, los docentes fueron seleccionados y supervisados por este mismo agente, el cual remuneraba sus servicios solo cuando conseguía acopiar los recursos suficientes para retribuir el trabajo de maestros y administrativos. Por alrededor de tres años no hubo un sueldo fijo. Ahora bien, la federación reconoció la antigüedad de los trabajadores desde el inicio del colegio, en 1973, por lo que pudieron ver recompensado su esfuerzo y sacrificio como una gratificación a largo plazo. De igual manera, los certificados expedidos como culminación de los estudios de bachillerato eran emitidos por la autoridad pública ${ }^{42}{ }^{43}$.

La entidad requería que el colegio continuara creciendo, por lo que se vio la necesidad, en 1978, de crear el Sistema de Enseñanza Abierta (SEA), con el propósito de atender a aquellos jóvenes que precisaran un modelo más flexible para poder concluir sus estudios preparatorios. De acuerdo con la rigidez imperante del sistema escolarizado del $\mathrm{COBACH}$, la sociedad requería una opción que se adaptara a las necesidades de los jóvenes trabajadores que no pudieran cumplir un horario estricto y les permitiera estudiar y realizar otras actividades simultáneamente. La primera ubicación del SEA fue en las calles $3^{\mathrm{a}}$ y Ojinaga hasta 1981, posteriormente en la Calle $2^{\text {a }}$ y Mina hasta 1983, luego en el Bulevar

\footnotetext{
40 Entrevista a López López.

41 Entrevista a Cendón Chávez.

42 Entrevista a Duarte Huerta.

43 Antonio Viñao Frago, Sistemas educativos, culturas escolares y reformas: continuidades y cambios (Madrid: Morata, 2002 ), 9.
} 
Díaz Ordaz y actualmente en la Ave. División del Norte 3707. El primer director fue el Lic. Florencio Estrada Loya ${ }^{44}$.

El éxito del colegio para la capital hizo eco en toda la entidad, lo que produjo que la sociedad demandara más espacios educativos, ampliación que se plasmó con una extensión del Plantel 2 en la ciudad de Chihuahua en 1979. Posteriormente, en 1982, esta extensión se convirtió en el Plantel 4, el cual estaba ubicado al norte de la ciudad de aquellos años, con su primer domicilio en el Edificio Caballeros de Colón. Para 1981 se inició la construcción de sus futuras y actuales instalaciones. En abril de 1982 fueron inauguradas por el director general nacional, el Mtro. José Ángel Vizcaíno Pérez, bajo la gestión del director C. P. Gonzalo Alberto Aguilera Gutiérrez, y los subdirectores Prof. Humberto Romero Sandoval del turno matutino y el Lic. Héctor Bustillos Mendoza del turno vespertino, con domicilio en la Calle Mtro. José Ángel Vizcaíno y Calle Pino, la cual fue renombrada en honor del director general nacional ${ }^{45}$.

De igual manera, la sociedad de Ciudad Juárez solicitó la apertura de escuelas con qué atender en esa ciudad de frontera la demanda de espacios educativos en el nivel medio superior. En 1982 inició la era del Colegio de Bachilleres para esa ciudad con la creación del Plantel 5, ubicado de manera transitoria en las instalaciones de la Escuela Primaria 21 de marzo, en Ave. Valle de Juárez. Luego el plantel se mudó para ocupar sus propias instalaciones con domicilio en la Calle Valentín Fuentes y la Calle Pedro Rosales de León, su ubicación actual. Se empezó a trabajar con siete grupos en cada turno, con alrededor de setecientos estudiantes, atendidos por treinta maestros, bajo la gestión del director, Prof. Ramón Ernesto Madrid Barroso, el subdirector del turno matutino, el Prof. Guillermo Valenzuela Gámez, y el subdirector del turno vespertino, el Ing. Rigoberto Garza Valdez ${ }^{46} 478$.

El buen desarrollo que llevaba el Plantel 5 en Ciudad Juárez motivó la fundación de otro recinto para la frontera. En 1984 se creó el Plantel 6, iniciando labores administrativas - registro de aspirantes, contrataciones de maestros, cursos propedéuticos - en las instalaciones de la Escuela Mariano Escobedo hasta septiembre del mismo año, cuando se iniciaron las actividades académicas en los antiguos edificios de la Universidad Regional del Norte (URN). Inició bajo la gestión del Lic. Jesús Terminal Soto como director y del Ing. Guadalupe Murga Meza como subdirector de ambos turnos. Al cabo de siete meses se reubicó a lo que sería su ubicación actual con domicilio en la Calle Faraday ${ }^{49}$.

La trayectoria que marcó el colegio como institución educativa, desde su creación hasta su consolidación, fue de prestigio, solidez y madurez. Se apuntaló

\footnotetext{
44 COBACH, Historia (Chihuahua: 2018), http://cobachih.edu.mx/Planteles/Sistema-de-Ense\%C3\%B1anza-Abierta/Chihuahua (8 de febrero, 2019).

45 Ibíd., http://cobachih.edu.mx/Planteles/Chihuahua/Plantel-4 (9 de febrero, 2019).

46 Ibíd., http://cobachih.edu.mx/Planteles/Ju\%C3\%A1rez/Plantel-5 (9 de febrero, 2019).

47 Entrevista a López López.

48 Hagelsieb Lerma, Inicio de la era del Colegio de Bachilleres, 60.

49 COBACH, Historia (Chihuahua: 2018), http://cobachih.edu.mx/Planteles/Ju\%C3\%A1rez/Plantel-6 (10 de febrero, 2019).
} 
como referente de excelencia académica en la capital del Estado y en la frontera, mediante sus esfuerzos por llevar una educación de calidad a los chihuahuenses para lograr con éxito el ingreso a la educación superior y formarlos como personas de bien. Todas estas premisas dieron pie para que, en 1985, se lograra la descentralización académica y funcional del $\mathrm{COBACH}$, iniciativa que fue impulsada por el titular del Ejecutivo Federal y la participación del secretario de Educación Pública, el gobernador del Estado, Lic. Saúl González Herrera y el director general del Colegio de Bachilleres de la Ciudad de México. Después el titular del Ejecutivo Estatal envió al Congreso del Estado la iniciativa de ley para la creación del Colegio de Bachilleres del Estado de Chihuahua como Organismo Público Descentralizado, con personalidad jurídica, competencia y patrimonios propios, el día 25 de diciembre de dicho año (imagen 7).

Imagen 7. Decreto de creación del COBACH como organismo público descentralizado.

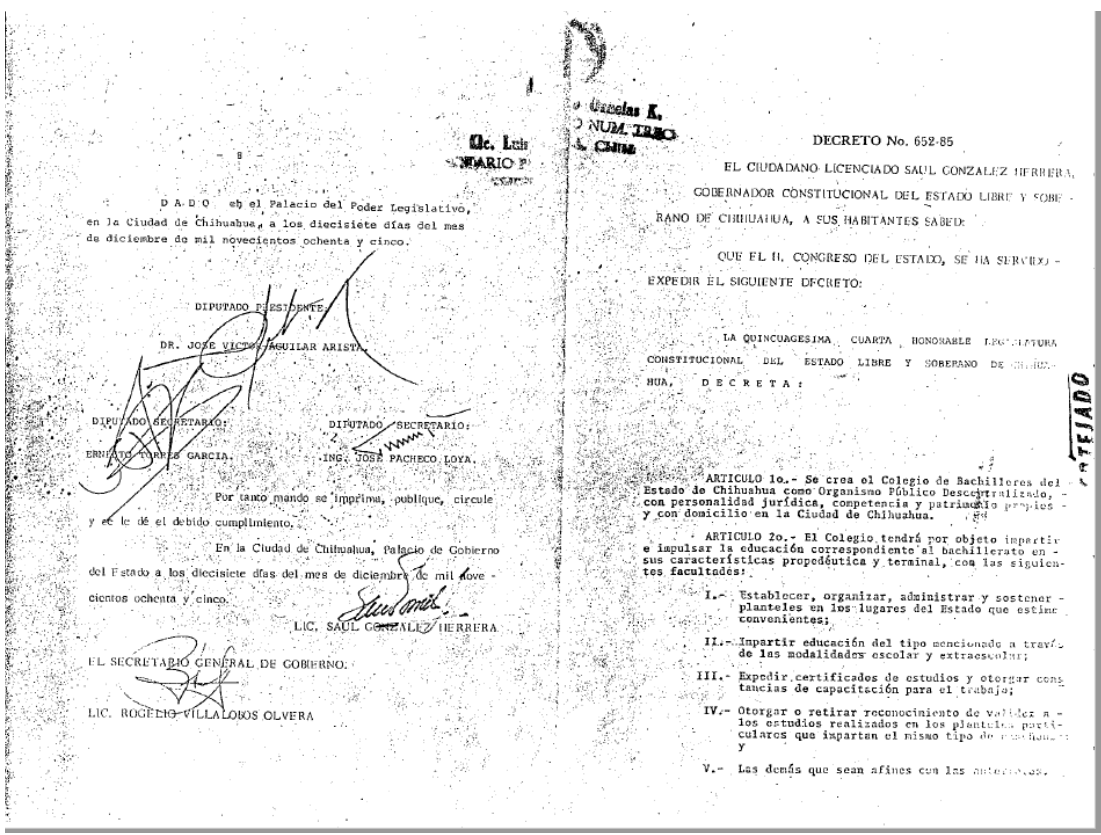

Fuente: Archivo jurídico del Colegio de Bachilleres del Estado de Chihuahua.

El colegio indudablemente comenzó una nueva etapa para la educación media en el Estado de Chihuahua, logró transformar la identidad juvenil bajo una cultura escolar cimentada en los valores del respeto, la obediencia, la responsabilidad y la disciplina. Esta cultura escolar que surge a raíz de la creación del $\mathrm{COBACH}$ se plasma en la sociedad para mantenerse durante toda la trayectoria de esta institución y para formar parte de la identidad de los jóvenes chihuahuenses de generación en generación. 


\section{CONCLUSIONES}

El periodo de análisis del acontecer del Colegio de Bachilleres para el Estado de Chihuahua, de 1973 a 1985, representó una etapa de transformación social para los jóvenes de nuestra entidad. Este cambio se vio escenificado en los espacios educativos que ocupó el $\mathrm{COBACH}$, por medio de "símbolos, estructuras, normas, pautas, tonos, estilos, modos, códigos, costumbres, tradiciones, formas de actuar, maneras de pensar, y de todos aquellos elementos que intervienen en la realidad cotidiana de nuestras escuelas" 50 .

El COBACH generó una renovación social que impulsó la educación de la entidad por las vías de la excelencia académica. La institución por medio de la transferencia de conocimientos y su ímpetu por la formación integral en valores de sus estudiantes, permeó más allá de los muros de sus recintos, adentrándose a través de sus procesos socio-educativos en la identidad de cada estudiante. El colegio logra su objetivo de reconocimiento social debido a que consigue responder a las necesidades sociales de la época. El control que ejerció sobre la juventud fue determinante para consolidarse como la primera opción de ingreso al nivel medio superior de aquellos años y en la actualidad.

La institución se apuntaló y se consolidó como un pilar para el sistema educativo chihuahuense, puesto que logró absorber en su totalidad el espacio social que había abandonado la Escuela Preparatoria para transfigurarlo en un espacio de excelencia académica, rigurosidad formativa y generador de una cultura escolar propia del colegio.

El aval del COBACH se evidencia en su continuidad y permanencia desde el año de su creación hasta la actualidad. Es manifiesto que los espacios educativos están en constante cambio, por lo que resulta imposible afirmar que los procesos actuales del colegio sigan siendo iguales a los de su inicio. Lo que sí se puede confirmar es que aun cuando la institución ha sufrido reformas a sus iniciales estatutos debido a la incorporación de los nuevos métodos de enseñanza y aprendizaje y para responder a las más actuales problemáticas educativas y sociales, en esencia la institución sigue con lealtad los objetivos con los cuales fue creada, tanto los explícitos como los ocultos.

\section{FUENTES}

Archivo Histórico de la Universidad Autónoma de Chihuahua (AHUACH). Chihuahua-México. Sección Educación, Fondo UACH.

Entrevista a Cendón Chávez, Gloria, Chihuahua, 14 de noviembre de 2018.

Entrevista a Duarte Huerta, Rodolfo, Chihuahua, 24 de septiembre de 2018.

50 Ramón López Martín, La escuela por dentro. Perspectivas de la cultura escolar en la España del siglo XX (Valencia: Publicacions de la Universitat de València, 2001), 20. 
Entrevista a García Quintana, Raymundo, Chihuahua, 14 de marzo de 2018. Entrevista a López López, Dora Elena, Chihuahua, 26 de octubre de 2018. Entrevista a Rico Bovio, Arturo, Chihuahua, 20 de febrero de 2018.

\section{REFERENCIAS}

Colegio de Bachilleres del Estado de Chihuahua. Historia. http://cobachih.edu.mx/Planteles/ Chihuahua/Plantel-1 (3 de febrero, 2019).

Colegio de Bachilleres del Estado de Chihuahua. Historia. http://cobachih.edu.mx/Planteles/ Chihuahua/Plantel-2 (4 de febrero, 2019).

Colegio de Bachilleres del Estado de Chihuahua. Historia. http://cobachih.edu.mx/Planteles/ Chihuahua/Plantel-3 (5 de febrero, 2019).

Colegio de Bachilleres del Estado de Chihuahua. Historia. http://cobachih.edu.mx/Planteles/ Chihuahua/Plantel-4 (9 de febrero, 2019).

Colegio de Bachilleres del Estado de Chihuahua. Historia. http://cobachih.edu.mx/Planteles/ Ju\%C3\%A1rez/Plantel-5 (9 de febrero, 2019).

Colegio de Bachilleres del Estado de Chihuahua. Historia. http://cobachih.edu.mx/Planteles/ Ju\%C3\%A1rez/Plantel-6 (10 de febrero, 2019).

Colegio de Bachilleres del Estado de Chihuahua. Historia. http://cobachih.edu.mx/Planteles/ Sistema-de-Ense\%C3\%B1anza-Abierta/Chihuahua (8 de febrero, 2019).

Colegio de Bachilleres del Estado de Chihuahua. Quiénes somos. http://www.cobachih.edu. $\mathrm{mx} /$ Colegio/Con\%C3\%B3cenos/Rese\%C3\%B1a-Hist\%C3\%B3rica (1 de febrero, 2019).

García, Agustín. "Colegio de Bachilleres, a 45 años de su fundación”. El Heraldo de Chihuahua, Chihuahua, 17 de abril, 2018.

Hagelsieb Lerma, Lombardo. Inicio de la era del Colegio de Bachilleres en nuestra frontera. Plantel No. 5. Ciudad Juárez: 2018.

López Martín, Ramón. La escuela por dentro: perspectivas de la cultura escolar en la España del siglo XX. Valencia: Publicacions de la Universitat de València, 2001.

Meneses Copete, Yeison Arcadio. “La etnoeducación afrocolombiana: conceptos, trabas, patriarcado y sexismo. A propósito de los 20 años de la Ley General de Educación 115 de 1994". Revista Historia de la Educación Latinoamericana, vol. 18, no. 27 (2016): 35-66.

Navarrete Cazales, Zaira. "¿Otra vez la identidad? Un concepto necesario pero imposible". Revista Mexicana de Investigación Educativa, vol. 20, no. 65 (2015): 461-479.

Neyra Galicia, Alma Rosa. “El bachillerato mexicano y la política educativa: desde sus inicios hasta la educación basada en competencias". Textual. Análisis del medio rural latinoamericano, no. 55 (2010): 63-82.

Ontiveros Juárez, Gerónimo, Francisco Alberto Pérez Piñón y Jesús Adolfo Trujillo Holguín. “El pensamiento crítico, democrático, humanista y el movimiento estudiantil de la UACH (1968-1974)", Millcayac. Revista Digital de Ciencias Sociales. vol. VII, no. 12 (2020): 348-368.

Pizarro Pizarro, Elías y Raúl Bustos González. “Educación y control político-social del Estado: visitadores de escuela en Tacna y Arica (1880-1900)". Revista Historia de la Educación Latinoamericana, vol. 17, no. 25 (2015): 125-138.

Salcido Sáenz, Marilyn Georgia y Jesús Adolfo Trujillo Holguín. “La construcción de procesos de identidad en las comunidades educativas, el caso del Colegio de Bachilleres del Estado de Chihuahua", Debates por la Historia, vol. VIII, no. 1 (2020): 243-266.

Sassen, Saskia. Territorio, autoridad y derechos: De los ensamblajes medievales a los ensamblajes globales. Buenos Aires: Katz, 2010.

Villa Lever, Lorenza. "La educación media superior: su construcción social desde el México independiente hasta nuestros días". En Los grandes problemas de México: VII Educación, editado por Alberto Arnaut y Silvia Giorguli. México: El Colegio de México, 2010, 271-311. 
Viñao Frago, Antonio. “Culturas escolares, reformas e innovaciones educativas”, Con-Ciencia Social, no. 5 (2001): 27-45.

Viñao Frago, Antonio. "Del espacio escolar y la escuela como lugar: propuestas y cuestiones", Historia de la Educación, no. 12-13 (1994): 17-74.

Viñao Frago, Antonio. “El espacio escolar: introducción”, Historia de la Educación,. no. 12 (1993): 11-16.

Viñao Frago, Antonio. Sistemas educativos, culturas escolares y reformas: continuidades y cambios. Madrid: Morata, 2002.

Cómo citar:

Salcido Sáenz, Marilyn Georgia; Trujillo Holguín, Jesús Adolfo. "El devenir de las instituciones educativas en el proceso de transformación social: el caso del Colegio de Bachilleres del Estado de Chihuahua, México (1973-1985)". Revista Historia de la Educación Latinoamericana. vol. 22 No. 34 (2020): 181-200 DOI: https://doi.org/10.19053/01227238.10899 (c) (1)@ Esta obra está bajo una licencia Creative Commons. Reconocimiento-No Comercial-Sin Obra Derivada 2.5 Colombia. 